\title{
Deformation Retracts of the Reissner-Nordstrom Spacetime and its Foldings
}

\author{
${ }^{1}$ A.E. El-Ahmady and ${ }^{2}$ A. Al-Rdade \\ ${ }^{1}$ Department of Mathematics, Faculty of Science, Taibah University, Madinah, Saudi Arabia \\ ${ }^{2}$ Department of Mathematics, Faculty of Science, Tanta University, Tanta, Egypt
}

Received 2012-09-16, Revised 2013-05-07; Accepted 2013-07-02

\begin{abstract}
Our aim in the present article is to introduce and study the relation between the deformation retract of the Reissner-Nordstrom spacetime $\mathrm{N}^{4}$ and the deformation retract of the tangent space $\mathrm{T}_{\mathrm{p}}\left(\mathrm{N}^{4}\right)$. Also, this relation discussed after and before the isometric and topological folding of $\mathrm{N}^{4}$ into itself. New types of conditional folding are presented. Some commutative diagrams are obtained.
\end{abstract}

Keywords: Retraction, Deformation Retracts, Folding, Reissner-Nordstrom Spacetime

\section{INTRODUCTION}

As is well known, the theory of foldings is always one of interesting topics in Euclidian and Non-Euclidian space and it has been investigated from the various viewpoints by many branches of topology and differential geometry (ElAhmady, 2013a; 2013b; 2013c; 2013d; 2013e).

Most folding problems are attractive from a pure mathematical standpoint, for the beauty of the problems themselves. The folding problems have close connections to important industrial applications Linkage folding has applications in robotics and hydraulic tube bending. Paper folding has application in sheet-metal bending, packaging and air-bag folding (El-Ahmady, 2012a; 2012b; 2011). Following the great Soviet geometer (El-Ahmady and AlRdade, 2013), also, used folding to solve difficult problems related to shell structures in civil engineering and aero space design, namely buckling instability (ElAhmady and Al-Hazmi, 2013). Isometric folding between two Riemannian manifold may be characterized as maps that send piecewise geodesic segments to a piecewise geodesic segments of the same length. For a topological folding the maps do not preserves lengths i.e., A map $\mathfrak{I}$ : $\mathrm{M} \rightarrow \mathrm{N}$, where $\mathrm{M}$ and $\mathrm{N}$ are $\mathrm{C}^{\infty}$-Riemannian manifolds of dimension $m, n$ respectively is said to be an isometric folding of $\mathrm{M}$ into $\mathrm{N}$, iff for any piecewise geodesic path $\gamma$ : $\mathrm{J} \rightarrow \mathrm{M}$, the induced path $\mathfrak{I} 0 \gamma: \mathrm{J} \rightarrow \mathrm{N}$ is a piecewise geodesic and of the same length as $\gamma$ (El-Ahmady and ElAraby, 2010). If $\mathfrak{I}$ does not preserve length, then $\mathfrak{I}$ is a topological folding. A subset A of a topological space $\mathrm{X}$ is called a retract of $X$ if there exists a continuous map $r$ : $\mathrm{X} \rightarrow \mathrm{A}$ such that $\mathrm{r}(\alpha)=\alpha, \forall \alpha \in \mathrm{A}$ where $\mathrm{A}$ is closed and $\mathrm{X}$ is open (Arkowitz, 2011; Banchoff and Lovett, 2010; ElAhmady, 2007a; 2007b, El-Ahmady, 2006; 2004a; 2004b). Also, let $X$ be a space and A a subspace. A map r: $\mathrm{X} \rightarrow \mathrm{A}$ such that $\mathrm{r}(\alpha)=\alpha$, for all $\alpha \in \mathrm{A}$, is called a retraction of $\mathrm{X}$ onto $\mathrm{A}$ and $\mathrm{A}$ is the called a retract of $\mathrm{X}$ (El-Ahmady and Shamara, 2001). This can be re stated as follows. If $\mathrm{i}$ : $\mathrm{A} \rightarrow \mathrm{X}$ is the inclusion map, then $\mathrm{r}: \mathrm{X} \rightarrow \mathrm{A}$ is a map such that $\mathrm{ri}=\mathrm{id}_{\mathrm{A}}$. If, in addition, ri $\mathrm{ri} \square \mathrm{id}_{\mathrm{x}}$ we call $\mathrm{r}$ a deformation retract and $\mathrm{A}$ a deformation retract of $\mathrm{X}$ (El-Ahmady, 1994). Another simple-but extremely useful-idea is that of a retract. If $\mathrm{A}, \mathrm{X} \subset \mathrm{M}$, then $\mathrm{A}$ is a retract of $\mathrm{X}$ if there is a commutative equation:

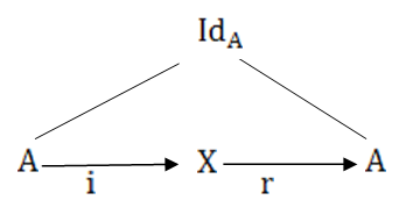
Corresponding Author: A.E. El-Ahmady, Department of Mathematics, Faculty of Science, Taibah University, Madinah, Saudi Arabia 
If $\mathrm{f}: \mathrm{A} \rightarrow \mathrm{B}$ and $\mathrm{g}: \mathrm{X} \rightarrow \mathrm{Y}$, then $\mathrm{f}$ is a retract of $\mathrm{g}$ if ri $=\mathrm{id}_{\mathrm{A}}$ and $\mathrm{js}=\mathrm{id}_{\mathrm{B}}$ (Naber, 2011; Reid and Szendroi, 2011; Arkowitz, 2011; Strom, 2011; Shick, 2007). At each point $\mathrm{p}$ of a complete Riemannian manifold $\mathrm{M}$, we define a mapping of the tangent space $T_{p}(M)$ at $p$ onto $\mathrm{M}$ in the following manner. If $\mathrm{X}$ is a tangent vector at $\mathrm{P}$ we draw a geodesic $g(t)$ starting at $P$ in the direction of $X$. If $X$ has length $\alpha$, then we map $X$ into the point $g(\alpha)$ of the geodesic. We denote this mapping by $\exp _{\mathrm{p}}$ : Tp $(\mathrm{M}) \rightarrow \mathrm{M}$, the map $\exp _{\mathrm{p}}$ is everywhere $\mathrm{C}^{\infty}$ and in a neighborhood of $\mathrm{p}$ in $\mathrm{M}$, it is a diffeomorphism (Kuhnel, 2006; Banchoff and Lovett, 2010).

\subsection{Main Results}

The Reissner-Nordström spacetime $\mathrm{N}^{4}$ is given by the following metric (El-Ahmady and Al-Rdade, 2013; Hartle, 2003; Griffiths and Podolsky, 2009; Straumann, 2003) Equation 1:

$\mathrm{ds}^{2}=-\left(1-\frac{2 \mathrm{~m}}{\mathrm{r}}+\frac{\mathrm{e}^{2}}{\mathrm{r}^{2}}\right) \mathrm{dt}^{2}+\left(1-\frac{2 \mathrm{~m}}{\mathrm{r}}+\frac{\mathrm{e}^{2}}{\mathrm{r}^{2}}\right)^{-1}$

$d r^{2}+r^{2}\left(d \theta^{2}+\sin ^{2} \theta d \phi^{2}\right)$

where, $m$ represents the gravitational mass and e the electric charge of the body.

The coordinates of Reissner-Nordstr Ö $\mathrm{m}$ spacetime $\mathrm{N}^{4}$ are given by Equation 2:

$$
\begin{aligned}
& \mathrm{x}_{1}^{2}=\mathrm{C}_{1-}\left(1-\frac{2 \mathrm{~m}}{\mathrm{r}}+\frac{\mathrm{e}^{2}}{\mathrm{r}^{2}}\right) \mathrm{t}^{2} \\
& \mathrm{x}_{2}^{2}=\mathrm{C}_{2+}\left(\mathrm{r}^{2}+4 \mathrm{mr}\right)+\left(4 \mathrm{~m}-\mathrm{e}^{2}\right) \operatorname{In}\left(\mathrm{r}^{2}-2 \mathrm{mr}+\mathrm{e}^{2}\right) \\
& +\left(8 \mathrm{~m}^{2}-4 \mathrm{e}^{2} \mathrm{~m}\right) \frac{1}{\sqrt{\mathrm{e}^{2}-\mathrm{m}^{2}}} \tan ^{-1} \frac{\mathrm{r}-\mathrm{m}}{\sqrt{\mathrm{e}^{2}-\mathrm{m}^{2}}} \\
& \mathrm{x}_{3}^{2}=\mathrm{C}_{3}+\mathrm{r}^{2} \theta^{2} \\
& \mathrm{x}_{4}^{2}=\mathrm{C}_{4+} \mathrm{r}^{2} \sin ^{2} \theta \phi^{2}
\end{aligned}
$$

where, $\mathrm{C}_{1}, \mathrm{C}_{2}, \mathrm{C}_{3}$ and $\mathrm{C}_{4}$ are the constant of integration .

The Reissner-Nordström space time $\mathrm{N}^{4}$ geodesic equations for the metric (1) are given by the following Equation 3-6:

$$
\begin{aligned}
& \frac{d u^{1}}{d \tau}+\frac{e^{2}-m r}{r\left(r^{2}-2 m r+e^{2}\right)}\left(u^{1}\right)^{2}-\frac{\left(r^{2}-2 m r+e^{2}\right)}{r}\left(u^{2}\right)^{2} \\
& -\frac{r^{2}-2 m r+e^{2}}{r} \sin ^{2} \theta\left(u^{3}\right)^{2}+ \\
& \frac{\left(\frac{m}{r^{2}}-\frac{e^{2}}{r^{3}}\right)\left(r^{2}-2 m r+e^{2}\right)}{r^{2}}\left(u^{4}\right)^{2}=0
\end{aligned}
$$

where, $\tau$ is an affine parameter. Suppose that $\gamma\left(\tau_{0}\right)=\left(\mathrm{r}_{0}, \theta_{0}, \frac{\pi}{2}, \mathrm{t}_{0}\right),(\mathrm{r}, \theta, \phi, \mathrm{t})$ corresponding $\left(\mathrm{u}^{1}, \mathrm{u}^{2}, \mathrm{u}^{3}, \mathrm{u}^{4}\right)$, for all $\tau$ where $\phi=\frac{\pi}{2}$. Then Equation 7:

$$
\frac{\mathrm{d} \phi}{\mathrm{d} \tau}=0=\mathrm{u}^{3}
$$

Under the condition $\mathrm{u}^{3}=0$ the above equations become Equation 8-11:

$$
\begin{aligned}
& \frac{\mathrm{du}^{1}}{\mathrm{~d} \tau}+\frac{\mathrm{e}^{2}-\mathrm{mr}}{\mathrm{r}\left(\mathrm{r}^{2}-2 \mathrm{mr}+\mathrm{e}^{2}\right)}\left(\mathrm{u}^{1}\right)^{2}-\frac{\left(\mathrm{r}^{2}-2 \mathrm{mr}+\mathrm{e}^{2}\right)}{\mathrm{r}} \\
& \left(\mathrm{u}^{2}\right)^{2}+\frac{\left(\frac{\mathrm{m}}{\mathrm{r}^{2}}-\frac{\mathrm{e}^{2}}{\mathrm{r}^{3}}\right)\left(\mathrm{r}^{2}-2 \mathrm{mr}+\mathrm{e}^{2}\right)}{}=0
\end{aligned}
$$

$\frac{d u^{3}}{d \tau}=0$

$\frac{d u^{4}}{d \tau}+\frac{2\left(-m+\frac{e^{2}}{r}\right)}{r^{2}-2 m r+e^{2}} u^{1} u^{4}=0$

Integrating Equation (9), we get Equation 12:

$$
\mu^{2}=\frac{\bar{\omega}_{2}}{\mathrm{r}^{2}}
$$

Also, integrating Equation (11), we get Equation 13:

$$
\mu^{4}=\frac{\bar{\omega}_{1} r^{2}}{r^{2}-2 m r+e^{2}}
$$


where, $\bar{\omega}_{1}$ and $\bar{\omega}_{2}$ are the constant of integrations. Substituting (7), (12) and (13) in (2), we get:

$$
\begin{gathered}
-\mathrm{x}_{1}^{2}+\mathrm{x}_{2}^{2}+\mathrm{x}_{3}^{2}+\mathrm{x}_{4}^{2}=\left(1-\frac{2 \mathrm{~m}}{\mathrm{r}}+\frac{\mathrm{e}^{2}}{\mathrm{r}^{2}}\right) \\
\left(\frac{\bar{\omega}_{1} \mathrm{r}^{2}}{\mathrm{r}^{2}-2 \mathrm{mr}+\mathrm{e}^{2}}\right)^{2}+\left(\mathrm{r}^{2}+4 \mathrm{mr}\right)+\left(4 \mathrm{~m}-\mathrm{e}^{2}\right) \\
\operatorname{In}\left(\mathrm{r}^{2}-2 \mathrm{mr}+\mathrm{e}^{2}\right)+\left(8 \mathrm{~m}^{2}-4 \mathrm{e}^{2} \mathrm{~m}\right) \frac{1}{\sqrt{\mathrm{e}^{2}-\mathrm{m}^{2}}} \tan ^{-1} \\
\frac{\mathrm{r}-\mathrm{m}}{\sqrt{\mathrm{e}^{2}-\mathrm{m}^{2}}}+\frac{\bar{\omega}_{2}^{2}}{\mathrm{r}^{2}}+\mathrm{H}_{1}
\end{gathered}
$$

Which is a hypersphere $\mathrm{S}_{1}^{3} \subset \mathrm{N}^{4}$ which is a geodesic retraction.

Again, substituting (7), (12) and (13) in (3), we get the following curves geodesic retraction $\mathrm{S}_{1} \subset \mathrm{N}^{4}$ $\left(u^{1}(\mu)\right)^{2}=\varpi_{1}^{2}+\left(k-\frac{\varpi_{2}^{2}}{r^{2}(\mu)}\right)\left(1-\frac{2 m}{r(\mu)}+\frac{e^{2}}{r^{2}(\mu)}\right)$ where $k=-1$ corresponds timelike geodesics and also $\mathrm{k}=0$ corresponds to null geodesics.

Then, the following theorem has been proved.

\section{Theorem 1}

Types of the geodesic retraction of ReissnerNordstrom spacetime $\mathrm{N}^{4}$ are hypersphere retraction and curves retraction.

\section{Theorem 2}

The deformation retract of $\left(\mathrm{N}^{4}-\left(\mathrm{p}_{1}, \mathrm{q}_{1}\right)\right)$ onto $\mathrm{S}_{1}^{3} \subset\left(\mathrm{N}^{4}-\left(\mathrm{p}_{1}, \mathrm{q}_{1}\right)\right)$ under the exponential map is an induced deformation retract of $\mathrm{T}_{\mathrm{p} 1} \quad\left(\mathrm{~N}^{4}\right)$ onto $\exp ^{-1}\left(\mathrm{~S}_{1}^{3}\right) \subset \mathrm{T}_{\mathrm{pl}}\left(\mathrm{N}^{4}-\mathrm{q}_{1}\right)$. Any isometric folding $\mathrm{F}: \mathrm{N}^{4} \rightarrow \mathrm{N}^{4}$ such that $\mathrm{F}\left(\mathrm{x}_{1}, \mathrm{x}_{2}, \mathrm{x}_{3}, \mathrm{x}_{4}\right)=\left(\mathrm{x}_{1}, \mathrm{x}_{2},\left|\mathrm{x}_{3}\right|, \mathrm{x}_{4}\right)$ induces the same deformation retract of $\mathrm{T}_{\mathrm{p} 1}\left(\mathrm{~N}^{4}\right)$ under the condition $\mathrm{x}_{3}=0$, which makes the equation:

$$
\begin{array}{ll}
\mathrm{D}_{\mathrm{p}_{1}}^{4}(\pi)-\mathrm{p}_{1} \stackrel{\mathrm{F}_{2}}{\longrightarrow} & \mathrm{D}_{\mathrm{p}_{1}}^{4}(\pi)-\mathrm{p}_{1} \\
\exp ^{-1} \uparrow & \uparrow \exp ^{-1} \\
\mathrm{~N}^{4}-\left(\mathrm{p}_{1}, \mathrm{q}_{1}\right) \stackrel{\mathrm{F}_{1}}{\longrightarrow} & \mathrm{N}^{4}-\left(\mathrm{p}_{1}, \mathrm{q}_{1}\right)
\end{array}
$$

Commutative, where $\left(\mathrm{D}_{\mathrm{p} 1}^{4}(\pi)-\mathrm{pl}\right)$ is an open ball of radius $\pi$ and center at $\mathrm{p}_{1}$.

\section{Proof}

The parametric equation of the Reissner-Nordström space time $\mathrm{N}^{4}$ is given:

$$
\begin{aligned}
& \xi=\left(\sqrt{ }\left(\mathrm{C}_{1}-\left(1-\frac{2 \mathrm{~m}}{\mathrm{r}(\mu)}+\frac{\mathrm{e}^{2}}{\mathrm{r}^{2}(\mu)}\right) \mathrm{t}^{2}(\mu)\right)\right. \\
& \sqrt{ }\left(C_{2+}\left(r^{2}(\mu)+4 m r(\mu)\right)+4\left(m-e^{2}\right)\right. \\
& \left.\operatorname{In}\left(\mathrm{r}^{2}(\mu)\right)-2 \operatorname{mr}(\mu)+\mathrm{e}^{2}\right) \\
& +\left(8 \mathrm{~m}^{2}-4 \mathrm{e}^{2} \mathrm{~m}\right) \\
& \frac{1}{\sqrt{\mathrm{e}^{2}-\mathrm{m}^{2}}} \tan ^{-1} \frac{\mathrm{r}(\mu)-\mathrm{m}}{\sqrt{\mathrm{e}^{2}-\mathrm{m}^{2}}} \\
& \sqrt{ }\left(\mathrm{C}_{3}+\mathrm{r}_{2}(\mu) \theta^{2}(\mu)\right) \\
& \left.\sqrt{C_{4+}} r^{2}(\mu) \sin ^{2} \theta(\mu) \phi^{2}(\mu)\right)
\end{aligned}
$$

By using lagrangian equations:

$$
\frac{\mathrm{d}}{\mathrm{ds}}\left(\frac{\partial \mathrm{T}}{\partial \mathrm{G}_{\mathrm{i}}}\right)-\left(\frac{\partial \mathrm{T}}{\partial \mathrm{G}_{\mathrm{i}}}\right)=0, \mathrm{i}=1,2,3,4
$$

where, $\mathrm{T}=\frac{1}{2} \mathrm{ds}^{2}$ we obtain the deformation retract of $\left(\mathrm{N}^{4}-\left(\mathrm{p}_{1}, \mathrm{q}_{1}\right)\right)$ given by:

$$
\begin{aligned}
& \mathrm{S}_{1}^{3}=\left(\sqrt{\mathrm{C}_{1}}, \sqrt{\mathrm{C}_{2}+\left(4 \mathrm{~m}-\mathrm{e}^{2}\right) \operatorname{In}\left(\mathrm{e}^{2}\right)+}\right. \\
& \left(8 \mathrm{~m}^{2}-4 \mathrm{e}^{2} \mathrm{~m}\right) \frac{1}{\sqrt{\mathrm{e}^{2}-\mathrm{m}^{2}}} \\
& \tan ^{-1} \frac{-\mathrm{m}}{\sqrt{\mathrm{e}^{2}-\mathrm{m}^{2}}}, \sqrt{\left.\mathrm{C}_{3} \sqrt{ } \mathrm{C}_{4}\right)}
\end{aligned}
$$

With retraction $\mathrm{R}_{1}, \mathrm{R}_{1}:\left(\mathrm{N}^{4}-\left(\mathrm{p}_{1}, \mathrm{q}_{1}\right)\right) \rightarrow \mathrm{S}_{1}^{3}$, then $\exp ^{-1}$ $\left(\mathrm{N}^{4}-\mathrm{q}_{1}\right)$ is an open ball $\mathrm{D}_{\mathrm{p} 1}^{\mathrm{n}}(\pi) \subset \mathrm{T}_{\mathrm{p} 1}(\mathrm{~N} 4)$. If $\mathrm{F}_{1}$ is a deformation retract of $\left(\mathrm{N}^{4}-\left(\mathrm{p}_{1}, \mathrm{q}_{1}\right)\right)$ onto a geodesic retraction $\mathrm{S}_{1}^{3}, \mathrm{~F}_{1}:\left\{\mathrm{N}^{4}-\left(\mathrm{p}_{1}, \mathrm{q}_{1}\right)\right\} \times \mathrm{I} \rightarrow_{\left\{\mathrm{N}^{4}-(\mathrm{pl} 1 \mathrm{q} 1)\right\}}$ such that:

$$
\begin{aligned}
& \mathrm{F}_{1}(\mathrm{x}, \mathrm{v})=(1-\mathrm{v})\left(\sqrt{(}\left(\mathrm{C}_{1}-\left(1-\frac{2 \mathrm{~m}}{\mathrm{r}(\mu)}+\frac{\mathrm{e}^{2}}{\mathrm{r}^{2}(\mu)}\right) \mathrm{t}^{2}(\mu)\right),\right. \\
& \sqrt{ }\left(\mathrm{C}_{2}+\left(\mathrm{r}^{2}(\mu)\right)+4 \mathrm{mr}(\mu)+4 \mathrm{~m}-\mathrm{e}^{2}\right) \operatorname{In}\left(\mathrm{r}^{2}(\mu)-2 \mathrm{mr}(\mu)+\mathrm{e}^{2}\right) \\
& +\left(8 \mathrm{~m}^{2}-4 \mathrm{e}^{2} \mathrm{~m}\right) \frac{1}{\sqrt{\mathrm{e}^{2}-\mathrm{m}^{2}}} \tan ^{-1} \frac{\mathrm{r}(\mu)-\mathrm{m}}{\left.\sqrt{\mathrm{e}^{2}-\mathrm{m}^{2}}\right)} \sqrt{ }\left(\mathrm{C}_{3}+\mathrm{r}^{2}(\mu) \theta^{2}(\mu)\right), \\
& \sqrt{ }\left(\mathrm{C}_{4+} \mathrm{r}^{2}(\mu) \sin ^{2}(\mu) \phi^{2}(\mu)\right)+\mathrm{v}\left(\sqrt{ }\left(\mathrm{C}_{2+}\right)\left(4 \mathrm{~m}-\mathrm{e}^{2}\right) \operatorname{In}\left(\mathrm{e}^{2}\right)+\right. \\
& \left.\left(8 \mathrm{~m}^{2}-4 \mathrm{e}^{2} \mathrm{~m}\right) \frac{1}{\sqrt{\mathrm{e}^{2}-\mathrm{m}^{2}}} \tan ^{-1} \frac{-\mathrm{m}}{\sqrt{\left.\mathrm{e}^{2}-\mathrm{m}^{2}\right)}} \sqrt{ } \mathrm{C}_{3} \sqrt{ } \mathrm{C}_{4}\right) \\
& \mathrm{F}_{1}(\mathrm{x}, 0)=\left(\sqrt{\left(\mathrm{C}_{1-}\right.}\left(1-\frac{2 \mathrm{~m}}{\mathrm{r}(\mu)}+\frac{\mathrm{e}^{2}}{\mathrm{r}^{2}(\mu)}\right) \mathrm{t}^{2}(\mu)\right) \sqrt{\left(\mathrm{C}_{2+}\right.}\left(\mathrm{r}^{2}(\mu)_{+4}\right. \\
& \operatorname{mr}(\mu))+\left(4 \mathrm{~m}_{-} \mathrm{e}^{2}\right) \operatorname{In}\left(\mathrm{r}^{2}(\mu)_{-2} \operatorname{mr}(\mu)+\mathrm{e}^{2}\right)+\left(8 \mathrm{~m}_{-4}^{2} \mathrm{e}^{2} \mathrm{~m}\right)
\end{aligned}
$$




$$
\begin{aligned}
& \left.\frac{1}{\sqrt{\mathrm{e}^{2}-\mathrm{m}^{2}}} \tan ^{-1} \frac{\mathrm{r}(\mu)-\mathrm{m}}{\sqrt{\left.\mathrm{e}^{2}-\mathrm{m}^{2}\right)}} \sqrt{\left(\mathrm{C}_{3}+\mathrm{r}^{2}(\mu)\right)} \theta^{2}(\mu)\right) \sqrt{\left(\mathrm{C}_{4+}\right.} \mathrm{r}^{2}(\mu) \text { and } \\
& \left.\sin ^{2} \theta(\mu) \phi^{2}(\mu)\right) \\
& \mathrm{F}_{1}(\mathrm{x}, 1)=\left(\sqrt{\mathrm{C}_{1}}, \sqrt{\left(\mathrm{C}_{2+}\right.}\left(4 \mathrm{~m}-\mathrm{e}^{2}\right) \operatorname{In}\left(\mathrm{e}^{2}\right) \sqrt{\mathrm{C}_{4}}\right)+\left(8 \mathrm{~m}^{2}-4 \mathrm{e}^{2} \mathrm{~m}\right) \\
& \frac{1}{\sqrt{\mathrm{e}^{2}-\mathrm{m}^{2}}} \tan ^{-1} \frac{-\mathrm{m}}{\sqrt{\left.\mathrm{e}^{2}-\mathrm{m}^{2}\right)}} \\
& \text { also } \quad \exp ^{-1}\left(\sqrt{\mathrm{C}_{1}} \sqrt{\left(\mathrm{C}_{2+}\right.}\left(4 \mathrm{~m}-\mathrm{e}^{2}\right) \operatorname{In}\left(\mathrm{e}^{2}\right)+\right. \\
& \quad\left(8 \mathrm{~m}^{2}-4 \mathrm{e}^{2} \mathrm{~m}\right) \frac{1}{\sqrt{\mathrm{e}^{2}-\mathrm{m}^{2}}} \tan ^{-1} \frac{-\mathrm{m}}{\sqrt{\mathrm{e}^{2}-\mathrm{m}^{2}}} \sqrt{\mathrm{C}_{3}}, \sqrt{\left.\mathrm{C}_{4}\right)} \text { is a }
\end{aligned}
$$

hypersphere $\mathrm{S}_{1}^{3} \subset \mathrm{T}_{\mathrm{p} 1}\left(\mathrm{~N}^{4}\right)$ of radius $\frac{\pi}{2}$ then there is an induced deformation retract of $\left(D_{\mathrm{pl}}^{4}(\pi)-\mathrm{p}_{1}\right)$ defined by $\mathrm{F}_{2}:\left\{\mathrm{D}_{\mathrm{pl}}^{4}(\pi)-\mathrm{p}_{1}\right\} \times \mathrm{I} \rightarrow\left\{\mathrm{D}_{\mathrm{pl}}^{4}(\pi)-\mathrm{p}_{1}\right\} \quad$ such that $\mathrm{F}_{2}\left(\left(\mathrm{x}_{1}, \mathrm{x}_{2}, \mathrm{x}_{3}, \mathrm{x}_{4}\right), \mathrm{v}\right)=\left(\mathrm{x}_{1}, \mathrm{x}_{2}, \mathrm{x}_{3}, \mathrm{x}_{4}\right)(1-\mathrm{v})+\frac{\pi\left(\mathrm{x}_{1}, \mathrm{x}_{2}, \mathrm{x}_{3}, \mathrm{x}_{4}\right)}{\mathrm{v} 2\left|\left(\mathrm{x}_{1}, \mathrm{x}_{2}, \mathrm{x}_{3}, \mathrm{x}_{4}\right)\right|}$ $\mathrm{F}_{2}\left(\left(\mathrm{x}_{1}, \mathrm{x}_{2}, \mathrm{x}_{3}, \mathrm{x}_{4}\right), 0\right)=\left(\mathrm{x}_{1}, \mathrm{x}_{2}, \mathrm{x}_{3}, \mathrm{x}_{4}\right)$ Where $\mathrm{F}_{2}\left(\left(\mathrm{x}_{1}, \mathrm{x}_{2}, \mathrm{x}_{3}, \mathrm{x}_{4}\right), 1\right)=\frac{\pi\left(\mathrm{x}_{1}, \mathrm{x}_{2}, \mathrm{x}_{3}, \mathrm{x}_{4}\right)}{2\left|\left(\mathrm{x}_{1}, \mathrm{x}_{2}, \mathrm{x}_{3}, \mathrm{x}_{4}\right)\right|}$ Which is $\exp ^{-1}\left\{\mathrm{~F}_{1}(\mathrm{x}, 1)\right\}=\mathrm{F}_{2}\left(\left(\mathrm{x}_{1}, \mathrm{x}_{2}, \mathrm{x}_{3}, \mathrm{x}_{4}\right), 1\right)=\mathrm{S}_{1}^{3}$

$\mathrm{S}_{1}^{3} \subset \mathrm{D}_{\mathrm{p} 1}^{4}(\pi)$, also $=\mathrm{F}_{2}\left\{\mathrm{D}_{\mathrm{p} 1}^{\mathrm{n}}(\pi)-\mathrm{p}_{1}\right\}=\mathrm{F}_{2}\left\{\exp ^{-1}\left(\mathrm{~N}^{4}-\mathrm{q}_{1}\right)\right\}$ $\Rightarrow \exp ^{-1} \mathrm{oF}_{1}=\mathrm{F}_{2} \mathrm{oexp}^{-1}$

and the following equation is commutative:

$$
\begin{array}{ll}
\left(\mathrm{D}_{\mathrm{p}_{1}}^{4}(\pi)-\mathrm{p}_{1}\right) \stackrel{\mathrm{F}_{2}}{\longrightarrow} & \left(\mathrm{D}_{\mathrm{p}_{1}}^{4}(\pi)-\mathrm{p}_{1}\right) \\
\exp ^{-1} \uparrow & \uparrow \exp ^{-1} \\
\left(\mathrm{~N}^{4}-\left(\mathrm{p}_{1}, \mathrm{q}_{1}\right)\right) \stackrel{\mathrm{F}_{1}}{\longrightarrow} & \left(\mathrm{N}^{4}-\left(\mathrm{p}_{1}, \mathrm{q}_{1}\right)\right)
\end{array}
$$

If $\mathrm{F}_{1}: \mathrm{N}^{4} \rightarrow \mathrm{N}^{4}$ is an isometric folding and any folding homeomorphic to this type of folding:

$$
\mathrm{F}_{1}\left(\mathrm{x}_{1}, \mathrm{x}_{2}, \mathrm{x}_{3}, \mathrm{x}_{4}\right)=\left(\mathrm{x}_{1}, \mathrm{x}_{2},\left|\mathrm{x}_{2}\right|, \mathrm{x}_{4}\right)
$$

Then $\mathrm{F}_{1}\left(\mathrm{~F}_{1}(\mathrm{x}, 1)\right)=\mathrm{F}_{1}(\mathrm{x}, 1)$, there is an induced isometric folding $\mathrm{F}_{2}:\left(\mathrm{D}_{\mathrm{pl}}^{4}(\pi)-\mathrm{p} 1\right) \rightarrow\left(\mathrm{D}_{\mathrm{pl}}^{4}(\pi)-\mathrm{p}_{1}\right)$ such that $\mathrm{F}_{2}:\left(\mathrm{S}_{1}^{3}\left(\frac{\pi}{2}\right)\right)=\mathrm{S}_{1}^{3}\left(\frac{\pi}{2}\right)$ i.e. $\mathrm{F}_{1}\left(\mathrm{~F}_{2}\left(\mathrm{x}_{1}, \mathrm{x}_{2}, \mathrm{x}_{3}, \mathrm{x}_{4}\right), 1\right)=$

$$
\mathrm{F}_{2}\left(\mathrm{x}_{1}, \mathrm{x}_{2}, \mathrm{x}_{3}, \mathrm{x}_{4}\right), \text { if } \mathrm{F}_{1}\left(\mathrm{~F}_{1}\right)=\mathrm{F}_{1}
$$

Let $\mathrm{F}_{\mu}$ is the set of all types of folding homeomorphic to $\mathrm{F}_{1}$ under the condition:

$$
\mathrm{F}_{1}\left(\mathrm{x}_{1}, \mathrm{x}_{2}, \mathrm{x}_{3}, \mathrm{x}_{4}\right)=\left(\mathrm{x}_{1}, \mathrm{x}_{2},\left|\mathrm{x}_{3}\right|, \mathrm{x}_{4}\right)
$$

Then the deformation retract of any $F \in F_{\mu}\left(N^{4}\right)$ is invariant, i.e.,

$F_{1}(f)=S_{1}^{3}$, the induced invariant deformations retract:

$$
\mathrm{F}_{2}\left\{\overline{\mathrm{F}}\left(\mathrm{D}_{\mathrm{p} 1}^{4}(\pi)-\mathrm{p} 1\right)\right\}=\mathrm{S}_{1}^{3}\left(\frac{\pi}{2}\right)
$$

\section{Theorem 3}

Under the condition $\mathrm{t}=\mathrm{e}=\mathrm{m}=0$, the deformation retract of $S_{1}^{2}-\left(p_{1}, q_{1}\right)$ onto $S_{1}^{1} \subset S_{1}^{2}-\left(p_{1}-q_{1}\right)$, under the exponential map is an induced deformation retract of $\mathrm{T}_{\mathrm{p} 1}\left(\mathrm{~S}_{1}^{2}\right) \quad$ onto $\exp ^{-1}\left(\mathrm{~S}_{1}^{1}\right) \subset \mathrm{T}_{\mathrm{pl}}\left(\mathrm{S}_{1}^{2}-\mathrm{q}_{1}\right)$. Any isometric folding $\mathrm{F}: \mathrm{S}_{1}^{2} \rightarrow \mathrm{S}_{1}^{2}$ such that $\mathrm{F}\left(\mathrm{x}_{1}, \mathrm{x}_{2}, \mathrm{x}_{3}\right)=\left(\mathrm{x}_{1}\left|\mathrm{x}_{2}\right| \mathrm{x}_{3}\right)$ induces the same deformation retract of $\mathrm{T}_{\mathrm{pl}}\left(\mathrm{S}_{1}^{2}\right)$, which makes the equation:

$$
\begin{array}{ll}
\mathrm{D}_{\mathrm{p}_{1}}^{2}(\pi)-\mathrm{p}_{1} \stackrel{\mathrm{F}_{2}}{\longrightarrow} & \mathrm{D}_{\mathrm{p}_{1}}^{4}(\pi)-\mathrm{p}_{1} \\
\exp ^{-1} \uparrow & \uparrow \exp ^{-1} \\
\mathrm{~S}_{1}^{2}-\left(\mathrm{p}_{1}, \mathrm{q}_{1}\right) \stackrel{\mathrm{F}_{1}}{\longrightarrow} \mathrm{S}_{1_{1}}^{2}-\left(\mathrm{p}_{1}, \mathrm{q}_{1}\right)
\end{array}
$$

Commutative, where $\mathrm{D}_{\mathrm{p} 1}^{2}(\pi)$ is an open ball of radius $\pi$ and of center at $\mathrm{p} 1$.

\section{Theorem 4}

Any isometric folding $\mathrm{F}: \mathrm{S}_{1}^{3} \subset \mathrm{N}^{4} \rightarrow \mathrm{S}_{1}^{3} \subset \mathrm{N}^{4}$ such that $\mathrm{F}(\mathrm{p})=\mathrm{p}, \mathrm{p}$ is any point on $\mathrm{S}_{1}^{3} \subset \mathrm{N}^{4}$. There is an induced isomtric folding of the tangent space $\mathrm{T}_{\mathrm{p} 1}\left(\mathrm{~S}_{1}^{3}\right)$ such that the following equation is commutative:

$$
\begin{array}{ccc}
\mathrm{T}_{\mathrm{p}_{1}}\left(\mathrm{~S}_{1}^{3}\right) \stackrel{\overline{\mathrm{F}}}{\longrightarrow} & \mathrm{T}_{\mathrm{p}_{1}}\left(\mathrm{~S}_{1}^{3}\right) \\
\exp ^{-1} \uparrow & & \uparrow \exp ^{-1} \\
\left(\mathrm{~S}_{1}^{2}-\mathrm{q}_{1}\right) & \stackrel{\mathrm{F}}{\longrightarrow} & \left(\mathrm{S}_{1}^{2}-\mathrm{q}_{1}\right)
\end{array}
$$

$\mathrm{q} 1$ is the conjugate point of p1, $p_{1}, q_{1} \in S_{1}^{3} \subset N^{4}$ i.e., $\exp ^{-1} o F=\bar{F}$ o $\exp ^{-1}$.

\section{Proof}

Since $\mathrm{q}_{1}$ is a conjugate point to $\mathrm{p}_{1}$, then $\exp ^{-1}$ : $\left(S_{1}^{3}-q_{1}\right) \rightarrow T_{p 1}\left(S_{1}^{3}\right)$ under this map $\left(S_{1}^{3}-q_{1}\right)$ mapped onto an open ball $\mathrm{D}_{\mathrm{p} 1}^{3} \subset \mathrm{T}_{\mathrm{p} 1}\left(\mathrm{~S}_{1}^{3}\right), \mathrm{p}_{1}$ is the center of the ball with radius $\pi$. 
Let $F:\left(\mathrm{S}_{1}^{3}-\mathrm{q}_{1}\right) \rightarrow\left(\mathrm{S}_{1}^{3}-\mathrm{q}_{1}\right)$ such that $\mathrm{F}\left(\mathrm{p}_{1}\right)=\mathrm{p}_{1}$ be an isometric folding, then there is an induced isometric folding $\overline{\mathrm{F}}$ such that:

$$
\overline{\mathrm{F}}: \mathrm{T}_{\mathrm{pl}}\left(\mathrm{S}_{1}^{3} \mathrm{t}\right) \rightarrow \mathrm{T}_{\mathrm{p} 1}\left(\mathrm{~S}_{1}^{3}\right)
$$

Let $\gamma$ be any curve in $\left(S_{1}^{3}-q_{1}\right)$ then $F(\gamma)=\dot{\gamma}$, since there is no conjugate point to $p_{1}$ on $\left(S_{1}^{3}-q_{1}\right)$, then $\exp ^{-1}$ $(\gamma)=\beta$, then $p_{1} \in \beta, p_{1}$ is the beginning of $\beta$, also $\exp ^{-1}(\dot{\gamma})=\dot{\beta}$. There is an induced isometric folding $\overline{\mathrm{F}}: \mathrm{T}_{\mathrm{pl}}\left(\mathrm{S}_{1}^{3}-\mathrm{q}_{1}\right) \rightarrow \mathrm{T}_{\mathrm{pl}}\left(\mathrm{S}_{1}^{3}-\mathrm{q}_{1}\right)$ such that:

$$
\begin{aligned}
& \overline{\mathrm{F}}(\beta)=\overline{\mathrm{F}}\left(\exp ^{-1}(\gamma)\right)=\alpha \\
& \exp ^{-1} \text { o } \mathrm{F}(\gamma) \_\exp ^{-1}(\dot{\gamma})+\dot{\beta} \\
& \exp ^{-1}\left(\mathrm{~F}\left(\mathrm{p}_{2}\right)\right)=\exp ^{-1}\left(\mathrm{P}_{3}\right)=\mathrm{P}_{3}^{\prime} \\
& \overline{\mathrm{F}}\left(\exp ^{-1}\left(\mathrm{p}_{2}\right)\right)
\end{aligned}
$$

Is the end of $\beta$ and the beginning point of $\alpha$ is the beginning point of $\beta$, the end point of $\alpha$ is the end point of $\beta$, then $\alpha=\beta$, i.e.:

$$
\exp ^{-1} \mathrm{oF}=\overline{\mathrm{F}} \mathrm{o} \exp ^{-1}
$$

\section{Theorem 5}

Under the condition $\mathrm{t}=\mathrm{e}=\mathrm{m}=0$, Any isometric folding $F: S_{1}^{2} \rightarrow S_{1}^{2}$ such that $F(p)=p, p$ is any point on $\mathrm{S}_{1}^{2}$. There is an induced isomtric folding of the tangent space $\mathrm{T}_{\mathrm{pl}} \mathrm{S}_{1}^{2}$ such that the following diagram is commutative:

$$
\begin{array}{ccc}
\mathrm{T}_{\mathrm{p}_{1}}\left(\mathrm{~S}_{1}^{2}\right) \stackrel{\overline{\mathrm{F}}}{\longrightarrow} & \mathrm{T}_{\mathrm{p}_{1}}\left(\mathrm{~S}_{1}^{2}\right) \\
\exp ^{-1} \uparrow & & \uparrow \exp ^{-1} \\
\left(\mathrm{~S}_{1}^{2}-\mathrm{q}_{1}\right) & \stackrel{\mathrm{F}}{\longrightarrow}\left(\mathrm{S}_{1}^{2}-\mathrm{q}_{1}\right)
\end{array}
$$

$\mathrm{q}_{1}$ is the conjugate point of $\mathrm{p}_{1}, \mathrm{p}_{1}, \mathrm{q}_{1} \in \mathrm{S}_{1}^{2}$ i.e.:

$$
\exp ^{-1} \mathrm{oF}_{1}=\mathrm{F}_{2} \mathrm{o} \exp ^{-1}
$$

\section{Theorem 6}

Under the conditions in theorem (2), if the following equation:

$$
\begin{array}{ll}
\left(\mathrm{D}_{\mathrm{p}_{1}}^{4}(\pi)-\mathrm{p}_{1}\right) \stackrel{\mathrm{F}_{2}}{\longrightarrow} & \left(\mathrm{D}_{\mathrm{p}_{1}}^{4}(\pi)-\mathrm{p}_{1}\right) \\
\exp ^{-1} \uparrow & \uparrow \exp ^{-1} \\
\left(\mathrm{~N}^{4}-\left(\mathrm{p}_{1}, \mathrm{q}_{1}\right)\right) \stackrel{\mathrm{F}_{1}}{\longrightarrow}\left(\mathrm{N}^{4}-\left(\mathrm{p}_{1}, \mathrm{q}_{1}\right)\right)
\end{array}
$$

Is commutative and $\mathrm{F}_{1}\left(\mathrm{~F}_{1}\right)=\mathrm{F}_{1}$, then the following equation is commutative:

$$
\begin{aligned}
& \left(\mathrm{D}_{\mathrm{p}_{1}}^{4}(\pi)-\mathrm{p}_{1}\right) \stackrel{\mathrm{F}_{2}}{\longrightarrow}\left(\mathrm{D}_{\mathrm{p}_{1}}^{4}(\pi)-\mathrm{p}_{1}\right) \\
& \exp ^{-1} \uparrow \\
& \left(\mathrm{Nexp}^{-1}\right. \\
& \left(\mathrm{N}^{4}-\left(\mathrm{p}_{1}, \mathrm{q}_{1}\right)\right) \stackrel{\mathrm{F}_{1}}{\longrightarrow}\left(\mathrm{N}^{4}-\left(\mathrm{p}_{1}, \mathrm{q}_{1}\right)\right)
\end{aligned}
$$

\section{Proof}

Since $\exp ^{-1} \circ F_{1}=F_{2} o \exp ^{-1}$, then:

$$
\mathrm{F}_{1}=\operatorname{exp~oF_{2}} \operatorname{oexp}^{-1}, \mathrm{~F}_{1}\left(\mathrm{~F}_{1}\right)=\mathrm{F}_{1}, \mathrm{~F}_{2}\left(\mathrm{~F}_{2}\right)=\mathrm{F}_{2}
$$

We get:

$$
\begin{aligned}
& \exp ^{-1} o_{1}=\exp ^{-1}\left(F_{1}\right)=\exp ^{-1} \\
& \left(\exp \text { o } F_{2} \exp ^{-1}\right)=F_{2} \text { o } \exp ^{-1}=F_{2} \text { o } \exp ^{-1}
\end{aligned}
$$

\section{CONCLUSION}

In this study we achieved the approval of the important of the curves and surface in ReissnerNordström spacetime $\mathrm{N}^{4}$ by using some geometrical transformations. The relations between folding, retractions, deformation retracts, limits of folding and limits of retractions of the curves and surface in the Reissner-Nordström spacetime $\mathrm{N}^{4}$ are discussed. New types of the tangent space $T_{p}\left(N^{4}\right)$ in Reissner-Nordström spacetime $\mathrm{N}^{4}$ are deduced.

\section{ACKNOWLEDGMENT}

The author is deeply indebted to the team work at the deanship of the scientific research, Taibah University for their valuable help and critical guidance and for facilitating many administrative procedures. This research work was financed supported by Grant no. 3066/1434 from the deanship of the scientific research at Taibah University, AlMadinah Al-Munawwarah, Saudi Arabia.

\section{REFERENCES}

Arkowitz, M., 2011. Introduction to Homotopy Theory. 1st Edn., Springer, New York, ISBN-10: 144197329X, pp: 344. 
Banchoff, T.F. and S.T. Lovett, 2010. Differential Geometry of Curves and Surfaces. 1st Edn., A.K. Peters, Natick, ISBN-10: 1568814569, pp: 331.

El-Ahmady, A.E. and A. Al-Rdade, 2013. A geometrical characterization of reissner-nordström spacetime and its retractions. Int. J. Applied Math. Stat., 36: 83-91.

El-Ahmady, A.E. and A. El-Araby, 2010. On fuzzy spheres in fuzzy minkowski space. Nuovo Cimento.

El-Ahmady, A.E. and H.M. Shamara, 2001. Fuzzy deformation retract of fuzzy horospheres. Ind. J. Pure Applied Math., 32: 1501-1506. DOI: 10.1007/BF02875737

El-Ahmady, A.E. and N. Al-Hazmi, 2013. Foldings and deformation retractions of hypercylinder. Ind. J. Sci. Technol., 6: 4084-4093.

El-Ahmady, A.E., 1994. The deformation retract and topological folding of Buchdahi space. Periodica Mathematica Hungarica, 28: 19-30.

El-Ahmady, A.E., 2004a. Fuzzy folding of fuzzy horocycle. Circolo Matematico Palermo, 53: 443450. DOI: $10.1007 / \mathrm{BF} 02875737$

El-Ahmady, A.E., 2004b. Fuzzy Lobachevskian space and its folding. J. Fuzzy Mathem., 12: 609-614.

El-Ahmady, A.E., 2006. Limits of fuzzy retractions of fuzzy hyperspheres and their foldings. Tamkang J. Mathem., 37: 47-55.

El-Ahmady, A.E., 2007a. Folding of fuzzy hypertori and their retractions. Proc. Math. Phys. Soc., 85: 1-10.

El-Ahmady, A.E., 2007b. The variation of the density functions on chaotic spheres in chaotic space-like Minkowski space time. Chaos, Solitons Fractals, 31: 1272-1278. DOI: 10.1016/j.chaos.2005.10.112

El-Ahmady, A.E., 2011. Retraction of chaotic black hole. J. Fuzzy Mthem., 19: 833-838.

El-Ahmady, A.E., 2012a. Retraction of null helix in Minkowski 3-space. Scientif. J. Applied Res., 1: 2833.

El-Ahmady, A.E., 2012b. Folding and unfolding of chaotic spheres in chaotic space-like Minkowski space-time. Scientif. J. Applied Res., 1: 34-43.
El-Ahmady, A.E., 2013a. Folding and fundamental groups of Buchdahi space. Ind. J. Sci. Technol., 6: 3940-3945.

El-Ahmady, A.E., 2013b. Fuzzy elastic Klein bottle and its retraction. Int. J. Applied Math. Stat., 42: 94-102.

El-Ahmady, A.E., 2013c. On the fundamental group and folding of Klein bottle. Int. J. Applied Math. Stat., 37: 56-64.

El-Ahmady, A.E., 2013d. On elastic Klein bottle and fundamental groups. Applied Math., 4: 499-504. DOI: $10.4236 / \mathrm{am} .2013 .43074$

El-Ahmady, A.E., 2013e. Folding and fundamental groups of flat Robertson-Walker Space. Ind. J. Sci. Technol., 6: 4235-4242.

Griffiths, J.B. and J. Podolsky, 2009. Exact Space-Times in Einstein's General Relativity. 1st Edn., Cambridge University Press, Cambridge, ISBN10: 1139481169 , pp: 525.

Hartle, J.B., 2003. Gravity, An Introduction to Einstein's General Relativity. 1st End., Pearson Education India, ISBN-10: 813170050X, pp: 656.

Kuhnel, W., 2006. Differential Geometry CurvesSurfaces-Manifolds. 2nd Edn., American Mathematical Soc, Providence, ISBN-10: 0821839888, pp: 380.

Naber, G.L., 2011. Topology, Geometry and Gauge Fields. 2nd Edn., Springer, New York, ISBN-10: 144197895X, pp: 419.

Reid, M. and B. Szendroi, 2011. Topology and Geometry. 1st Edn., American Mathematical Soc, ISBN-10: 0821856502, pp: 263.

Shick, P.L., 2007. Topology: Point-Set and Geometry. 1st Edn., Wiley-Interscience, New York, ISBN-10: 0470096055, pp: 271.

Straumann, N., 2003. General Relativity with Application to Astrophysics. 1st Edn., Springer, New York, ISBN-10: 038740628X, pp: 312.

Strom, J., 2011. Modern Classical Homotopy Theory. 1st Edn., American Mathematical Society, Providence, ISBN-10: 0821884298, pp: 835. 